\title{
Theory of second harmonic generation in a chirped 2D nonlinear optical superlattice under nonlinear Raman-Nath diffraction
}

\author{
ANDREY M. VYUNISHEV ${ }^{1,2, *}$, VASILY G. ARKHIPKIN ${ }^{1,3}$, AND ANATOLY S. CHIRKIN ${ }^{4}$ \\ ${ }^{1}$ L.V. Kirensky Institute of Physics, Krasnoyarsk 660036, Russia \\ ${ }^{2}$ Department of Photonics and Laser Technology, Siberian Federal University, Krasnoyarsk 660079, Russia \\ ${ }^{3}$ Laboratory for Nonlinear Optics and Spectroscopy, Siberian Federal University, Krasnoyarsk 660079, Russia \\ ${ }^{4}$ Faculty of Physics and International Laser Center, M. V. Lomonosov Moscow State University, Moscow 119992, Russia \\ ${ }^{*}$ Corresponding author: vyunishev@iph.krasn.ru \\ Compiled August 28, 2015
}

\begin{abstract}
We analyze second harmonic generation (SHG) in a two dimensional nonlinear optical superlattice (NLOS) with its modulation period being chirped in the propagation direction and constant in the transverse direction. This results in efficient multiple SHG via nonlinear Raman-Nath diffraction. We obtain exact analytical expressions for a SH amplitude generated in chirped 2D NLOSs and for its quasi-phasematching bandwidth. The results of analytical calculations are in excellent agreement with the numerical ones. We show that the process is robust to angular deviations of NLOS and it can be applied to enable tunable and broadband frequency conversion. ๑ 2015 Optical Society of America
\end{abstract}

OCIS codes: (190.2620) Harmonic generation and mixing; (190.4223) Nonlinear wave mixing; (190.4420) Nonlinear optics, transverse effects in.

http://dx.doi.org/10.1364/ao.XX.XXXXXX

\section{INTRODUCTION}

One of the challenges of nonlinear optics is to create a new family of multichannel nonlinear optical converters that offer ultra wide spectral tunability and large angular aperture. The most promising phenomenon, which can be used for these proposes, is nonlinear Raman-Nath diffraction (NRND) in onedimensional nonlinear optical superlattices (NLOSs) [1-6]. The main advantage of this phenomenon is the ability to emit multiple second harmonic ( $\mathrm{SH}$ ) beams at characteristic angles relative to the incoming fundamental frequency (FF) beam. The disadvantage of this process is oscillation of the $\mathrm{SH}$ amplitude along the propagation direction because of the phase mismatch between FF and $\mathrm{SH}$ waves. To solve this problem twodimensional (2D) NLOSs were proposed [7, 8]. The authors of [9] experimentally studied second harmonic generation (SHG) in an almost periodic 2D NLOS. The periodicity of the superlattice in the propagation direction allows us to realize SHG under quasi-phase-matching for a discrete number of wavelengths, which are different for different NRND orders. In [8], a method of superposition of nonlinear susceptibility modulation is applied for designing 2D NLOSs to overcome this circumstance. At the same time, the 2D NLOSs described in [8,9] are characterized by narrow spectral and angular acceptances.
On the contrary, 2D NLOSs with random spatial fluctuations of the nonlinear coefficient in the propagation direction possess a wide range of reciprocal superlattice vectors (RSV) to match appropriate FF and $\mathrm{SH}$ wavevectors [7]. In this case, enhanced SHG can be achieved in wide spectral and angular ranges. Alternatively, chirped spatial modulation of the nonlinear coefficient of superlattices can help solve the problem. This is the most feasible technique to obtain a broader RSV spectrum. Contrary to the cases of angular- and quasi-phasematching, the spectral response of chirped structures increases with propagation length. Chirped 1D NLOSs have been shown to be promising for compressing and stretching optical pulses [10] and for broadband harmonic [11] and biphoton [12,13] generation. Chirped structures are also considered to be promising for adiabatic frequency conversion with efficiency as high as possible. The basic concept of and recent advances in adiabatic frequency conversion are reviewed in [14]. However, in our opinion an exact analytical expression to characterize SHG in chirped lattices has not been found yet.

In the present work we study second harmonic generation in a 2D nonlinear optical superlattice. We designed a rectangular nonlinear optical superlattice such that its modulation period is chirped in the propagation direction and periodical modulation is in the transverse direction. We obtain an exact analyti- 
cal expression for the $\mathrm{SH}$ amplitude generated in a chirped 2D NLOS. The results are found to be in excellent agreement with numerical calculations. The employed approach ensures efficient multiple frequency generation via nonlinear Raman-Nath diffraction. These results open up new possibilities to create multichannel nonlinear optical converters for a wide range of applications.

\section{THEORETICAL MODEL}

We begin our analysis on the second harmonic generation by considering propagation of the fundamental wave through a chirped 2D nonlinear optical superlattice presented in Fig. 1 (a). It is assumed that the nonlinear susceptibility of the superlattice is modulated periodically along the transverse direction (axis $x$ ) and represents a chirped rectangular function along the propagation direction (axis $y$ ). This configuration allows us to match FF $\left(\mathbf{k}_{1}\right)$ and $\mathrm{SH}\left(\mathbf{k}_{2}\right)$ wavevectors by appropriate RSVs as shown in Fig. 1 (b). In particular, the linear dependence of RSV along the propagation direction can be used to compensate wavevector mismatches between longitudinal components $\left(\mathbf{q}_{y}=\mathbf{k}_{\|}\right)$ while the discreet set of RSV in transverse direction produces series of NRND orders $\left(\mathbf{q}_{x}=\mathbf{k}_{\perp}\right)$. It can be mathematically expressed as momentum conservation conditions in projections onto the corresponding axes:

$$
\begin{gathered}
\Delta k_{\perp}=\left|\mathbf{k}_{2}\right| \sin (\theta)-2\left|\mathbf{k}_{1}\right| \sin (\gamma)=m\left|\mathbf{q}_{x}\right|, \\
\Delta k_{\|}=\left|\mathbf{k}_{2}\right| \cos (\theta)-2\left|\mathbf{k}_{1}\right| \cos (\gamma)=n\left|\mathbf{q}_{y}\right|,
\end{gathered}
$$

here $\gamma$ and $\theta$ are the inner FF and SH propagation angles, respectively; $m$ is the NRND order and $n$ is the effective QPM order. Eq. (1) is known as the nonlinear Raman-Nath condition that can be represented in the most convenient form

$$
\sin \theta_{m}=\frac{2\left|\mathbf{k}_{1}\right| \sin (\gamma)+m\left|\mathbf{q}_{x}\right|}{\left|\mathbf{k}_{2}\right|}
$$

Eq. (2) describes the condition for Cerenkov nonlinear diffraction [15-22]. To satisfy this condition for a set of NRND orders, linearly chirped (gradually stepped) spatial modulation of the sign of nonlinear susceptibility can be applied. (a)

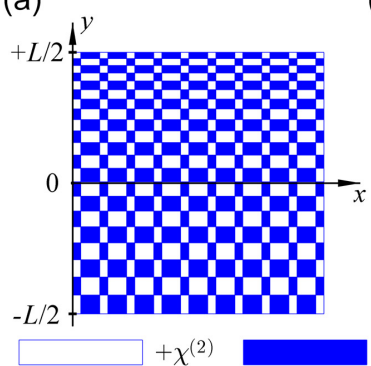

(b)

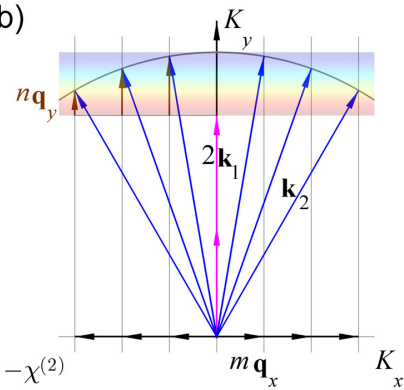

Fig. 1. Spatial variance of the sign of nonlinear susceptibility over a chirped superlattice (a) and the vectorial phase matching diagram (b). The rainbow region illustrates the range of appropriate values of reciprocal superlattice vectors.

The second order nonlinear susceptibility of the superlattice can be represented as a binary function of two spatial coordinates $g(x, y)$ taking the values \pm 1 over the structure, while the refractive index is supposed to be homogeneous. It is assumed that the conversion efficiency is low and undepleted field approximation can be applied. Moreover, the process under consideration occurs within a narrow angular range, i.e. $\mathrm{SH}$ propagation angles $\theta_{m}$ are small enough to satisfy $\sin \theta_{m} / \theta_{m} \approx 1$. This means that the problem under study can be considered in paraxial approximation. Then, given these assumptions, the process of $\mathrm{SH}$ generation can be described by the equation

$$
\left(\frac{\partial}{\partial y}+\frac{i}{2 k_{2}} \Delta_{\perp}\right) A(\mathbf{r}, y)=\Gamma g(x, y) F^{2}(\mathbf{r}) e^{i \Delta k y}
$$

where $\Gamma=-2 i \pi k_{2} \chi^{(2)} A_{1}^{2} / n_{2}^{2}, A_{1}$ and $A$ are the complex FF and $\mathrm{SH}$ field amplitudes, respectively, $\chi^{(2)}$ is the second order nonlinear susceptibility, $\Delta k=k_{2}-2 k_{1}$ is the wavevector mismatch, $\Delta_{\perp}=\left(\partial^{2} / \partial x^{2}+\partial^{2} / \partial z^{2}\right)$ is the transverse Laplacian, $n_{2}$ denotes the refractive index at the $\mathrm{SH}$ frequency, $F(\mathbf{r})=\exp \left(-\mathbf{r}^{2} / a^{2}\right)$ is the transverse distribution of the fundamental beam intensity, $r^{2}=x^{2}+z^{2}$, and $a$ is the spot radius.

To solve Eq. (4), it is easy to represent the amplitude $A(\mathbf{r}, y)$ as a Fourier spectrum:

$$
A(\mathbf{K}, y)=\iint A(\mathbf{r}, y) e^{i \mathbf{K r}} d x d z
$$

here $K^{2}=K_{x}^{2}+K_{z}^{2}$.

The limits of integration are from $-\infty$ to $\infty$ and and will be omitted in our further discussion. Then Eq. (4) can be rewritten as

$$
\left(\frac{\partial}{\partial y}-\frac{i \mathbf{K}^{2}}{2 k_{2}}\right) A(\mathbf{K}, y)=\Gamma \iint g(x, y) F^{2}(\mathbf{r}) e^{i \Delta k y} e^{i \mathbf{K r}} d x d z .
$$

We define the function $g(x, y)$ as a product of two functions $g(x, y)=\xi(x) \eta(y)$, which are responsible for the nonlinear susceptibility modulation along respective directions. The spatial dependence of the nonlinear coefficient along $x$ direction can be expressed as following Fourier series

$$
\xi(x)=\sum_{m=0, \pm 1, \ldots} \xi_{m} e^{i m q_{x} x}
$$

where $q_{x}=2 \pi / \Lambda$ is the primary reciprocal superlattice vector (spatial frequency), $\Lambda$ is the modulation period. The Fourier coefficients $\xi_{m}$ for a periodic rectangular function with the duty cycle $D$ take the form $\xi_{m}=2 D-1$ if $m=0$ and $\xi_{m}=$ $(2 / \pi m) \sin (\pi m D)$ otherwise. The duty cycle value defined as a ratio of the relevant domain thickness to the modulation period influences the magnitude of the corresponding Fourier coefficient and the strength of respective nonlinear diffraction order.

Let us define the spatial modulation of the nonlinear coefficient along $y$ direction as

$$
\eta(y)=\operatorname{sgn}(\sin (K(y) y))
$$

where the local spatial frequency $K(y)=q_{y}(1+\beta y)$ is a slowly varying function of the coordinate $y \in[-L / 2, L / 2], q_{y}$ is the primary spatial frequency in the center of the superlattice at $y=$ 0 and $\beta$ is the rate of frequency increase or the chirp parameter. Note that $\beta=0$ corresponds to the periodic structure.

The signum function can be represented in an integral form

$$
\operatorname{sgn}(f(y))=(2 / \pi) \int_{0}^{\infty} \frac{\sin (u f(y))}{u} d u .
$$


Substituting Eq. (8) into Eq. (9) yields

$\sin (u \sin (K(y) y))$

$$
=2 \sum_{p=0}^{\infty} J_{2 p+1}(u) \sin ((2 p+1) K(y) y),
$$

where $J_{2 p+1}(u)$ is the Bessel functions of real argument.

Taking into account the integral relation

$$
\int_{0}^{\infty} u^{-1} J_{2 p+1}(u) d u=\frac{1}{2 p+1}
$$

we arrive at [23]:

$$
\eta(y)=(4 / \pi) \sum_{p=0}^{\infty} \frac{1}{2 p+1} \sin ((2 p+1) K(y) y) .
$$

As expected from Eq. (12) the spatial frequency chirp appears in all orders of spatial modulation of nonlinearity.
Integrating Eq. (6) over the transverse coordinates, which account for Eq. (7), and assuming Eq. (12), we obtain

$$
\begin{aligned}
& A\left(K_{x}, y\right) \exp \left(-i\left(\left(K_{x}^{2}+K_{z}^{2}\right) / 2 k_{2}\right) y\right)=(4 \alpha / \pi) \\
& \left.\quad \times \sum_{p=0,1,2, \ldots} \frac{1}{2 p+1} \int \sin (Q(1+\beta y) y) \exp (i \Delta \tilde{k} y) d y\right) .
\end{aligned}
$$

Here, $\alpha=\pi a^{2} \Gamma / 2, \Delta \tilde{k}=\Delta k-K_{x}^{2} / 2 k_{2}, Q(p)=-(2 p+1) q_{y}$ is the reciprocal superlattice vector for the central frequency in the center of the lattice and $2 p+1$ can be treated as the QPM order, the function $R\left(K_{x}\right)=\sum_{m} \xi_{m} \exp \left(-a^{2}\left(\left(K_{x}+m q_{x}\right)^{2}+K_{z}^{2}\right) / 8\right)$ is associated with phase matching of the transverse components of FF and SH wavevectors. The solution of Eq. (13) is a $\mathrm{SH}$ amplitude generated in a 2D superlattice (compare to [12]):

$$
\begin{aligned}
A\left(K_{x}, K_{z}, L\right)=( & \alpha / \pi)(-1)^{3 / 4} \exp \left(i \frac{\left(K_{x}^{2}+K_{z}^{2}\right)}{2 k_{2}} L\right) \sum_{p=0,1,2, \ldots} \frac{1}{2 p+1} R\left(K_{x}\right) \sqrt{\frac{\pi}{\beta Q}} \\
& \times\left\{i \exp \left(-i \frac{(\Delta \tilde{k}+Q)^{2}}{4 \beta Q}\right)\left[\operatorname{erfi}\left(\frac{(1+i)(\Delta \tilde{k}+Q+\beta Q L)}{2 \sqrt{2 \beta Q}}\right)-\operatorname{erfi}\left(\frac{(1+i)(\Delta \tilde{k}+Q-\beta Q L)}{2 \sqrt{2 \beta Q}}\right)\right]\right. \\
& \left.+\exp \left(i \frac{(\Delta \tilde{k}-Q)^{2}}{4 \beta Q}\right)\left[\operatorname{erfi}\left(\frac{(1-i)(\Delta \tilde{k}-Q+\beta Q L)}{2 \sqrt{2 \beta Q}}\right)-\operatorname{erfi}\left(\frac{(1-i)(\Delta \tilde{k}-Q-\beta Q L)}{2 \sqrt{2 \beta Q}}\right)\right]\right\} .
\end{aligned}
$$

Here, $\operatorname{erfi}(z)=-i \operatorname{erf}(i z)=2 / \sqrt{\pi} \int_{0}^{z} \exp \left(t^{2}\right) d t$ is an imaginary error function. Note, that summation in Eq. (14) is performed over positive integers and the first term is responsible for the quasi-phase-matching when the condition $\Delta \tilde{k}+Q \pm$ $\beta Q L=0$ is satisfied, while the second one contributes to the process if $\Delta \tilde{k}-Q \pm \beta Q L=0$. The case under study is $\Delta \tilde{k}+Q \pm \beta Q L=0$ and the second term in Eq. (14) can be ignored.

Analyzing Eq. (14) we obtained a simple formula for the SHG spectral bandwidth under NRND in a chirped 2D superlattice

$$
\Delta \lambda_{2}(\beta, m) \simeq\left|\frac{2 \beta Q L}{\frac{d}{d \lambda_{2}}(\Delta \tilde{k})}\right|=\left|\frac{2 \beta Q L}{\frac{d}{d \lambda_{2}}\left(\Delta k-\left(m q_{x}\right)^{2} / 2 k_{2}\right)}\right| .
$$

where $\lambda_{2}$ is the SH wavelength.

Usually a large spectral bandwidth means wide angular acceptance of the nonlinear converter. In our case, a spectral component being in the center of the spectral bandwidth at normal incidence will be efficiently converted to $\mathrm{SH}$ when propagating into the structure at a critical angle

$$
\gamma_{c r}(\beta, m) \simeq \pm \arccos \left(\frac{1}{2 k_{1}}\left[k_{2}-\frac{\left(m q_{x}\right)^{2}}{2 k_{2}}+\left(1+\frac{\beta L}{2}\right) Q\right]\right)
$$

The solution for chirped superlattices is significantly different from the one corresponding to a periodic lattice. For further investigation, we need to find a solution for a periodic structure. In this case spatial modulation of the nonlinear coefficient along the propagation direction can be expressed in the form of Eq. (7)

$$
\eta(y)=\sum_{n=0, \pm 1, \ldots} \eta_{n} e^{i n q_{y} y}
$$

where $\eta_{n}$ are the Fourier coefficients.

Under this assumption, the solution of Eq. (6) is

$$
\begin{aligned}
& A\left(K_{x}, L\right)=\alpha L R\left(K_{x}\right) \\
& \times \sum_{n=0, \pm 1, \ldots} \eta_{n} \exp \left(i L \frac{\left(K_{x}^{2}+K_{z}^{2}\right)}{2 k_{2}}\right) \operatorname{sinc}\left(\frac{L(\Delta \tilde{k}+Q)}{2}\right) .
\end{aligned}
$$

Here, $Q=n q_{y}$. Eq. (18) is consistent with the results of paper [24], where Cerenkov nonlinear diffraction in a 2D NLOS was considered.

In the case of a lattice with periodic modulation the QPM SHG bandwidth equals

$$
\Delta \lambda_{2}(m) \simeq\left|\frac{0.886 \pi}{L \frac{d}{d \lambda_{2}}(\Delta \tilde{k})}\right|
$$

While the spatial frequency takes the values in accordance with Eq. (1), i.e. $K_{x}(\theta, \gamma)=k_{2} \sin (\theta)-2 k_{1} \sin (\gamma)$, the wavevector mismatch in longitudinal direction obeys Eq. (2), i.e. $\Delta \tilde{k}(\theta, \gamma)=k_{2} \cos (\theta)-2 k_{1} \cos (\gamma)$. Indeed, if $K_{x}^{2} / 2 k_{2} \ll k_{2}$ in Eq. (12) we obtain $k_{2} \cos (\theta) \simeq k_{2}-K_{x}^{2} / 2 k_{2}$.

\section{RESULTS AND DISCUSSIONS}

It is obvious that nonlinear Raman-Nath diffraction should have a different effect in different 2D NLOS. In this context, we consider spectral and spatial characteristics acquired by $\mathrm{SH}$ under nonlinear Raman-Nath diffraction in periodic and chirped 2D NLOS. We will analyze the influence of spatial parameters of the lattices on the $\mathrm{SH}$ characteristics.

We choose lithium niobate as a nonlinear medium and consider propagation and interaction of extraordinary waves. The 
fundamental radiation was chosen to be linearly polarized along $z$ axis so FF and $\mathrm{SH}$ waves were coupled by the relevant nonlinear coefficient of lithium niobate $\chi^{(2)}=2 d_{33}$. The principal refractive indexes of lithium niobate were approximated by the Sellmeier coefficients from Ref. [25]. First, we calculate spectral characteristics of $\mathrm{SH}$ generated in periodic and chirped 2D NLOS. Eq. (18) was used to calculate the spectral intensity $S\left(K_{x}, L\right)=\left|A\left(K_{x}, L\right)\right|^{2}$ in a periodic lattice. The following parameters were chosen for the calculations: central fundamental wavelength $800 \mathrm{~nm}$, beam size $80 \mu \mathrm{m}$ (FWHM), thickness of the superlattice $L=5 \mathrm{~mm}$, NRND order $m=0$, QPM order $n=3$ (RSV $Q=3 q_{y}, \Lambda=7.73 \mu \mathrm{m}$ and the respective Fourier coefficient $\eta_{n=3}=-2 /(3 \pi)$ ). The calculated spectral dependence of the $\mathrm{SH}$ intensity is shown in Fig. 2. For the case of a periodic structure the spectral dependence has the shape of a typical $\operatorname{sinc}^{2}(x)$-function, $0.05 \mathrm{~nm}$-wide (FWHM) and located at half of the central fundamental wavelength. Using Eq. (14), we calculated SHG in a positively chirped (up-chirped) superlattice of the same length. We assume that the $3^{\text {rd }}$-order QPM SHG $(2 p+1=3)$ for the $0^{\text {th }}$ NRND order $(m=0)$ is realized in the center of the superlattice $(y=0)$. The chirp parameter is taken to be $\beta=4 \mathrm{~m}^{-1}$ since the spatial frequency changes from 0.805 to $0.821 \mu^{-1}$. This means that the effective period varies from 7.81 to $7.66 \mu \mathrm{m}$ throughout the superlattice. Note, that the layer thicknesses fit the hyperbolic law for spatial frequency. One can see from Fig. 2 that the spectral response of the chirped structure is wider than the one corresponding to the periodic structure. The spectral dependence has ripples resulting from oscillations of the erfi-functions in Eq. (14). In order to smooth the spectral ripples, an apodization technique [26] may be additionally employed to design the structure, which however is beyond our consideration. The results obtained can be generalized for the case of broadband up-conversion in chirped 1D NLOSs.

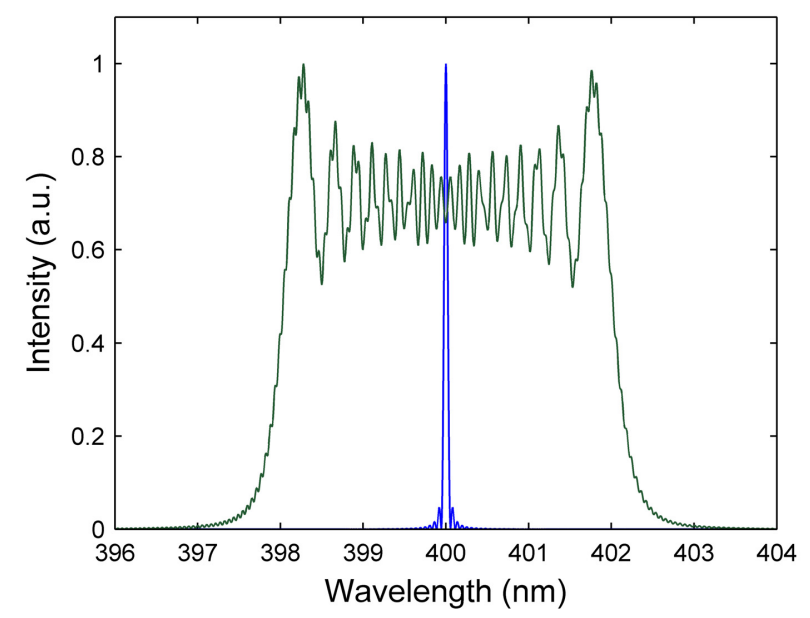

Fig. 2. SH spectra generated in periodic (blue) and chirped (green) 2D nonlinear superlattices for the zeroth order NRND.

Again, using Eq. (18) we calculate spatial distribution of the $\mathrm{SH}$ spectral intensity for NRND in periodic 2D NLOS. We chose the duty cycle $D_{x}=0.74$ to obtain comparable Fourier coefficients for the first three NRND orders $\left(\xi_{m=0}=0.48, \xi_{m=1} \approx\right.$ $0.46, \xi_{m=2} \approx-0.32$ ). The result of these calculations is presented in Fig. 3 (a). It is seen that the NRND orders experience a spectral shift towards shorter wavelengths as the NRND order grows [9]. This means that using quasi-monochromatic radiation will result in inefficient SHG for all NRND orders except for one whose longitudinal phase-matching condition satisfied. On the contrary, the chirped structure exhibits wider spectral responses for all NRND orders as shown in Fig. 3 (b). The spectral ranges for different orders may overlap ensuring efficient NRND for a set of orders.
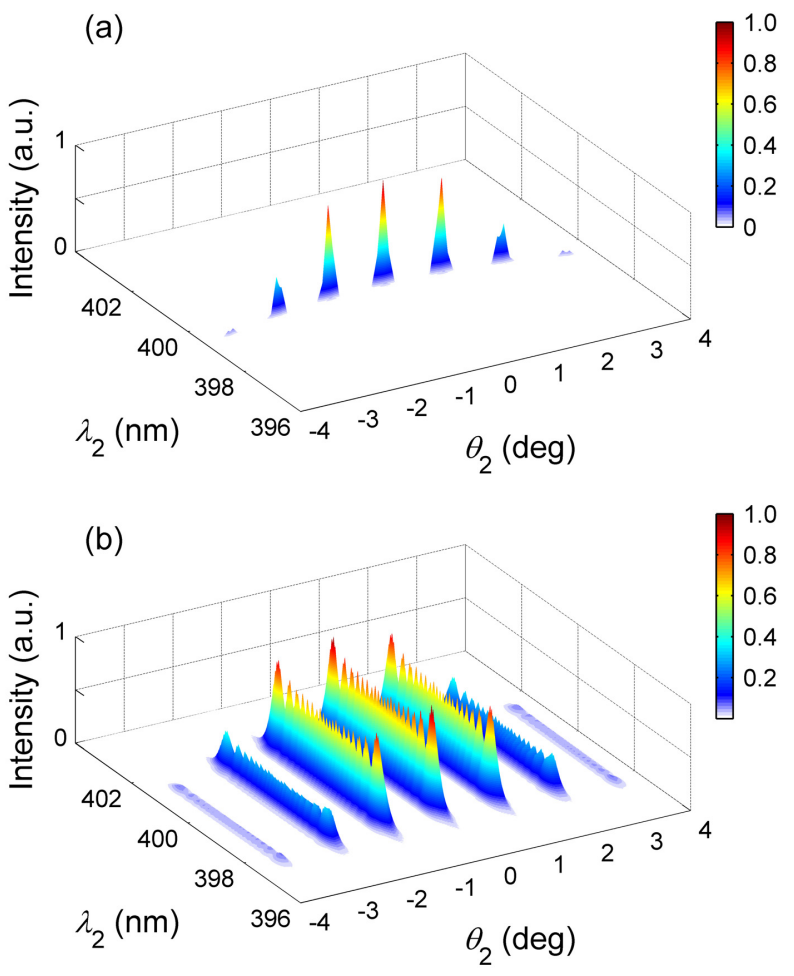

Fig. 3. Angular distribution of the spectral intensity of SH radiation in periodic (a) and chirped (b) 2D nonlinear optical superlattices.

For a certain NRND order, the SH spectral components evolve along the structure as shown in Fig. 4. It is seen that the spectral response of the structure is linearly distributed over the structure. As the fundamental radiation propagates through the structure, new spectral components are involved in the process. When the $\mathrm{SH}$ intensity reaches its maximum, weak intensity oscillations appear. The scale of oscillations decreases in the propagation direction, which can be explained by spatial dependence of the phase mismatch in the longitudinal direction. Namely, the farther away from the region of exact QPM, the smaller the scale of oscillation. These oscillations are accompanied by minor reduction in the intensity along the propagation direction. Moreover, the spectral width grows along the propagation length, while the details of $\mathrm{SH}$ spectra become smaller. From Fig. 4 we evaluate the spectral width to be $0.8 \mathrm{~nm} \cdot \mathrm{mm}^{-1}$. Note that changing the sign of the chirp parameter results in mirror inversion of the calculated dependence in the spectral range relative to the central $\mathrm{SH}$ wavelength. This can be simply achieved by inverting the coordinates $y \rightarrow-y$.

Next, we study the influence of the chirp parameter on $\mathrm{SH}$ spectral characteristics. In Fig. 5 (a), the calculated dependence is shown in the range of relatively small values of the chirp parameter $\beta=0.1-5 \mathrm{~m}^{-1}$. These results are represented as a 


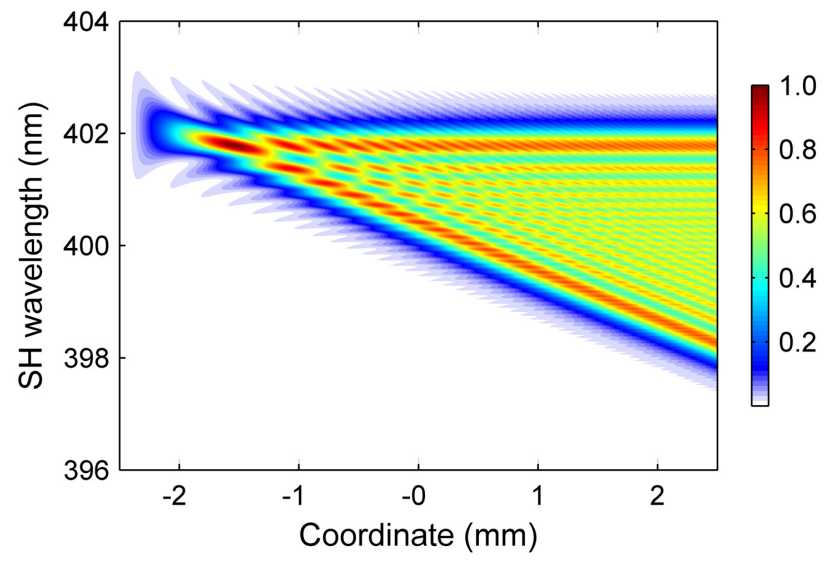

Fig. 4. Evolution of the SH spectral intensity along the propagation direction in a chirped superlattice for zero order NRND $\left(\beta=4 \mathrm{~m}^{-1}\right)$.

product $\beta S\left(K_{x}, L\right)$ because the spectral intensity is an inverse function of the chirp parameter and hence the spectral intensity decays rapidly (see Eq. (14)) unlike the dependence on the coordinate (Fig. 4). In some sense, these dependences are similar, i.e. the dependence in Fig. 5 behaves in the same manner as the dependence on the propagation coordinate (Fig. 4). Fig. 5 (b) shows distribution of the spectral intensity for periodic and chirped 2D NLOS for selected chirp parameters $\beta=4,20,40$ $\mathrm{m}^{-1}$. On the one hand, increasing the chirp parameter leads to spectral broadening. From Fig. 5 (b), the corresponding spectral bandwidths are 4.0, 21.0, $43.2 \mathrm{~nm}$. Eq. (15) gives the following spectral bandwidths $\Delta \lambda_{2}=4.4,21.8,43.6 \mathrm{~nm}$. On the other hand, the external critical angle calculated using Eq. (15) is $4.6 \mathrm{deg}$ for $m=0$ and $\beta=4 \mathrm{~m}^{-1}$. Therefore, the choice of the chirp parameter is a trade-off between a wide spectral (angular) bandwidth and high SHG efficiency. Eq. (14) allows us to find the optimal value of the chirp parameter to design 2D NLOS enabling efficient conversion of ultrabroadband laser radiation.

We numerically verified Eq. (14) using the approach described in [8]. This approach takes into account contributions from a sequence of layers in arbitrary structured rectangular 2D NLOS. Fig. 6 illustrates the results of numerical calculations of the spectral response in a chirped lattice in comparison with the results obtained from the analytical expression (Eq. (14)). These dependences coincide with each other in a wide range of intensities. We can conclude that Eq. (14) provides high accuracy of calculations.

\section{CONCLUSION}

We have elaborated the theory of second harmonic generation under nonlinear Raman-Nath diffraction in a two dimensional nonlinear optical superlattice characterized by chirped modulation of the nonlinear susceptibility in longitudinal direction. It is shown that a chirped $2 \mathrm{D}$ superlattice supports broadband multiple second harmonic generation via nonlinear Raman-Nath diffraction. An expression for the spectral bandwidth of quasi-phase matched SH generation has been obtained. The approach developed can be easily generalized to the case of supercontinuum radiation [27]. These results may inspire a wide range of applications in laser wavelength multiplexing (a)
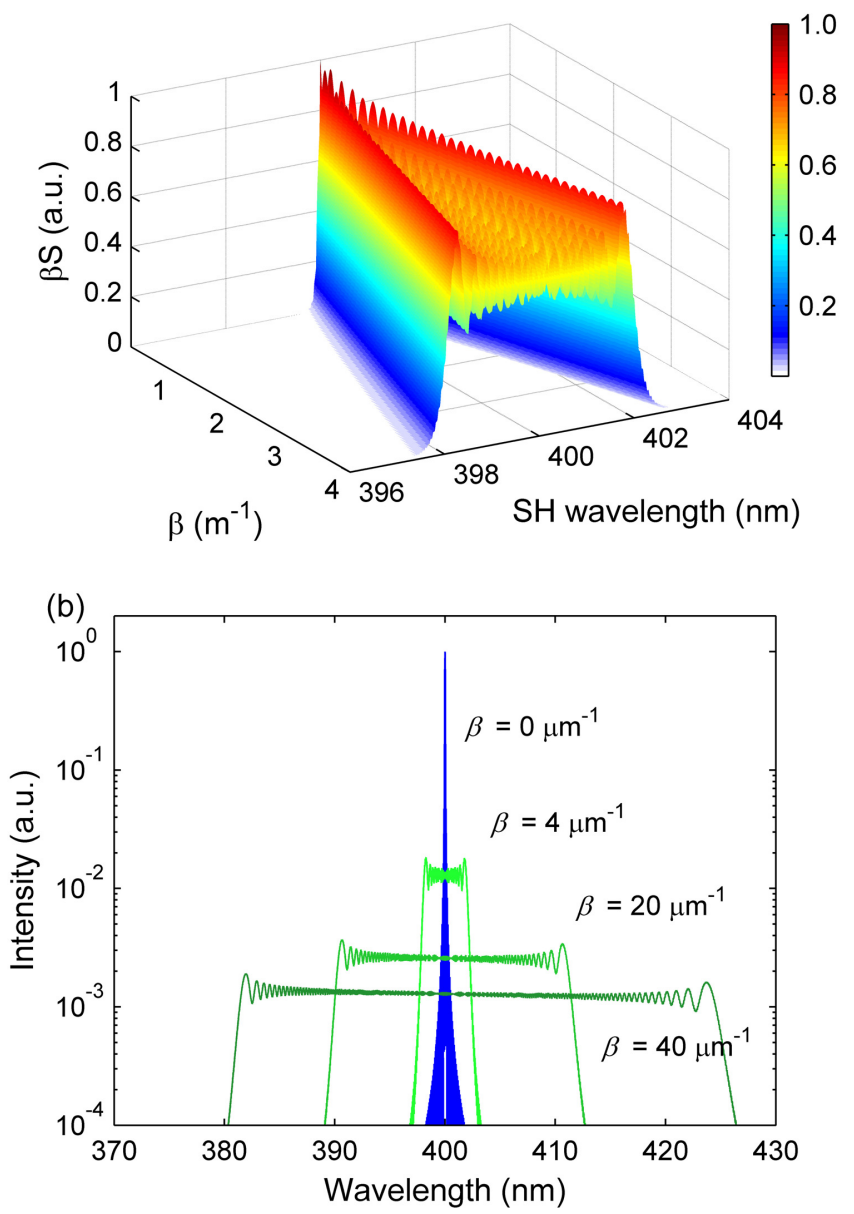

Fig. 5. Dependence of the SH spectral intensity on the chirp parameter (a) and a set of $\mathrm{SH}$ spectra for characteristic values of the chirp parameter $\left(\beta=4,20,40 \mathrm{~m}^{-1}\right)(\mathrm{b})$.

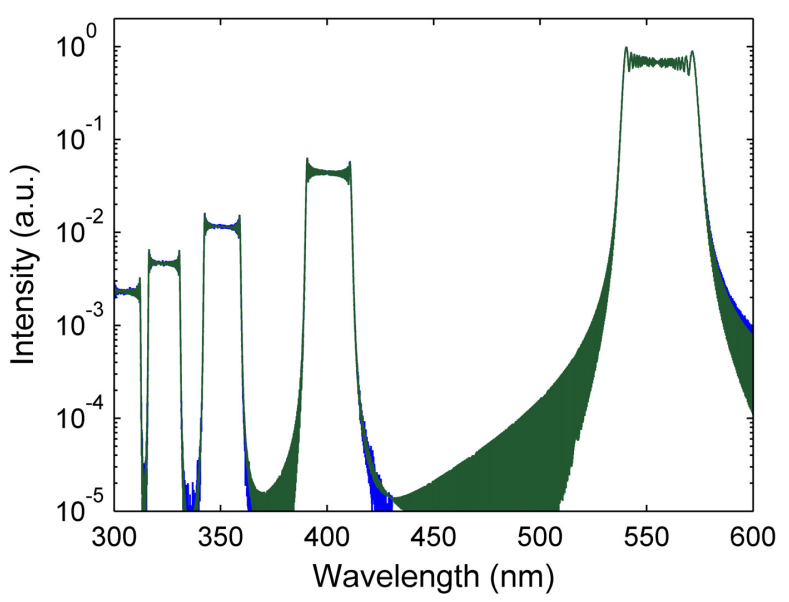

Fig. 6. Spectral response of chirped structures calculated numerically (blue) and analytically $\left(\beta=20 \mathrm{~m}^{-1}\right)$ (green). The selected bands correspond to the following QPM orders $p=1,3,5,7$ (from right to left). 
and telecommunications.

\section{FUNDING}

Council of the President of the Russian Federation (MK2908.2015.2); Russian Foundation for Basic Research (RFBR) (1502-03838).

\section{REFERENCES}

1. S. M. Saltiel, D. N. Neshev, R. Fischer, W. Krolikowski, A. Arie, and Y. S. Kivshar, "Generation of second-harmonic conical waves via nonlinear bragg diffraction," Phys. Rev. Lett. 100, 103902 (2008).

2. S. M. Saltiel, D. N. Neshev, W. Krolikowski, A. Arie, O. Bang, and Y. S. Kivshar, "Multiorder nonlinear diffraction in frequency doubling processes," Opt. Lett. 34, 848-850 (2009).

3. K. Kalinowski, P. Roedig, Y. Sheng, M. Ayoub, J. Imbrock, C. Denz, and W. Krolikowski, "Enhanced Cerenkov second-harmonic emission in nonlinear photonic structures," Opt. Lett. 37, 1832-1834 (2012).

4. Y. Sheng, Q. Kong, W. Wang, K. Kalinowski, and W. Krolikowski, "Theoretical investigations of nonlinear Raman-Nath diffraction in the frequency doubling process," Journal of Physics B: Atomic, Molecular and Optical Physics 45, 055401 (2012).

5. Y. Chen, W. Dang, Y. Zheng, X. Chen, and X. Deng, "Spatial modulation of second-harmonic generation via nonlinear Raman-Nath diffraction in an aperiodically poled lithium tantalite," Opt. Lett. 38, 2298-2300 (2013).

6. A. M. Vyunishev, V. V. Slabko, I. S. Baturin, A. R. Akhmatkhanov, and V. Y. Shur, "Nonlinear Raman-Nath diffraction of femtosecond laser pulses," Opt. Lett. 39, 4231-4234 (2014).

7. W. Wang, Y. Sheng, V. Roppo, Z. Chen, X. Niu, and W. Krolikowski, "Enhancement of nonlinear Raman-Nath diffraction in two-dimensional optical superlattice," Opt. Express 21, 18671-18679 (2013).

8. A. M. Vyunishev and A. S. Chirkin, "Multiple quasi-phase-matching in nonlinear Raman-Nath diffraction," Opt. Lett. 40, 1314-1317 (2015).

9. A. M. Vyunishev, V. G. Arkhipkin, V. V. Slabko, I. S. Baturin, A. R. Akhmatkhanov, V. Y. Shur, and A. S. Chirkin, "Nonlinear Raman-Nath diffraction of femtosecond laser pulses in a 2D nonlinear photonic crystal," Opt. Lett. 40, 4002-4005 (2015).

10. M. A. Arbore, A. Galvanauskas, D. Harter, M. H. Chou, and M. M. Fejer, "Engineerable compression of ultrashort pulses by use of secondharmonic generation in chirped-period-poled lithium niobate," Opt. Lett. 22, 1341-1343 (1997).

11. B.-Q. Chen, M.-L. Ren, R.-J. Liu, C. Zhang, Y. Sheng, B.-Q. Ma, and Z.-Y. Li, "Engineerable compression of ultrashort pulses by use of second-harmonic generation in chirped-period-poled lithium niobate," Light. Sci. Appl. 3, 1-6 (2014).

12. S. E. Harris, "Chirp and compress: Toward single-cycle biphotons," Phys. Rev. Lett. 98, 063602 (2007).

13. M. B. Nasr, S. Carrasco, B. E. A. Saleh, A. V. Sergienko, M. C. Teich, J. P. Torres, L. Torner, D. S. Hum, and M. M. Fejer, "Ultrabroadband biphotons generated via chirped quasi-phase-matched optical parametric down-conversion," Phys. Rev. Lett. 100, 183601 (2008).

14. H. Suchowski, G. Porat, and A. Arie, "Adiabatic processes in frequency conversion," Laser and Photonics Reviews 8, 333-367 (2014).

15. R. Fischer, S. M. Saltiel, D. N. Neshev, W. Krolikowski, and Y. S. Kivshar, "Broadband femtosecond frequency doubling in random media," Applied Physics Letters 89, 191105 (2006).

16. A. S. Aleksandrovsky, A. M. Vyunishev, A. I. Zaitsev, A. V. Zamkov, and V. G. Arkhipkin, "Detection of randomized nonlinear photonic crystal structure in a non-ferroelectric crystal," Journal of Optics A: Pure and Applied Optics 9, S334 (2007).

17. I. Shutov, I. Ozheredov, A. Shumitski, and A. Chirkin, "Second harmonic generation by femtosecond laser pulses in the Laue scheme," Optics and Spectroscopy 105, 79-84 (2008).

18. P. Molina, M. d. I. O. Ramírez, and L. E. Bausá, "Strontium barium niobate as a multifunctional two-dimensional nonlinear "photonic glass"," Advanced Functional Materials 18, 709-715 (2008).
19. S. Saltiel, Y. Sheng, N. Voloch-Bloch, D. Neshev, W. Krolikowski, A. Arie, K. Koynov, and Y. S. Kivshar, "Cerenkov-type second-harmonic generation in two-dimensional nonlinear photonic structures," Quantum Electronics, IEEE Journal of 45, 1465-1472 (2009).

20. X. Deng, H. Ren, H. Lao, and X. Chen, "Research on Cherenkov second-harmonic generation in periodically poled lithium niobate by femtosecond pulses," J. Opt. Soc. Am. B 27, 1475-1480 (2010).

21. A. M. Vyunishev, A. S. Aleksandrovsky, A. I. Zaitsev, and V. V. Slabko, "Čerenkov nonlinear diffraction in random nonlinear photonic crystal of strontium tetraborate," Applied Physics Letters 101, 211114 (2012).

22. A. M. Vyunishev, A. S. Aleksandrovsky, A. I. Zaitsev, and V. V. Slabko, "Cerenkov nonlinear diffraction of femtosecond pulses," J. Opt. Soc. Am. B 30, 2014-2021 (2013).

23. A. S. Chirkin and I. V. Shutov, "Parametric amplification of light waves at low-frequency pumping in aperiodic nonlinear photonic crystals," Journal of Experimental and Theoretical Physics 109, 547-556 (2009).

24. Y. Sheng, V. Roppo, Q. Kong, K. Kalinowski, Q. Wang, C. Cojocaru, J. Trull, and W. Krolikowski, "Tailoring Cerenkov second-harmonic generation in bulk nonlinear photonic crystal," Opt. Lett. 36, 2593-2595 (2011).

25. D. H. Jundt, "Temperature-dependent sellmeier equation for the index of refraction, $n_{e}$, in congruent lithium niobate," Opt. Lett. 22, 1553-1555 (1997).

26. C. R. Phillips, C. Langrock, D. Chang, Y. W. Lin, L. Gallmann, and M. M. Fejer, "Apodization of chirped quasi-phasematching devices," J. Opt. Soc. Am. B 30, 1551-1568 (2013).

27. K. Krupa, A. Tonello, D. Pagnoux, A. Labruyère, V. Couderc, B. M. Shalaby, F. Baronio, "Infrared supercontinuum frequency doubling via nonlinear Raman-Nath and Cerenkov scattering processes," Opt. Commun. 355, 114-118 (2015). 